\title{
SACMI Electronic Olfactory System Based on Semiconducting Metal Oxides ${ }^{\dagger}$
}

\author{
Marco Marzocchi * and Oliviero Ossani \\ SACMI Imola S.C., via Selice Provinciale, 17/A, 40026 Imola (BO), Italy; oliviero.ossani@sacmi.it \\ * Correspondence: marco.marzocchi@sacmi.it \\ + Presented at the 8th GOSPEL Workshop. Gas Sensors Based on Semiconducting Metal Oxides: Basic \\ Understanding \& Application Fields, Ferrara, Italy, 20-21 June 2019.
}

Published: 19 June 2019

Electronic Olfactory Systems, also called Electronic Noses, are instruments designed to mimic the sense of smell. This is obtained by using an array of different gas sensors, whose signals are collected and elaborated by a processing unit; the measured signals are then compared to a predetermined odour training set, in order to obtain odour recognition and quantification.

In SACMI Electronic Noses, an array of six different semiconducting metal oxide gas sensors is used as the sensing element (Figure 1). These sensors are obtained by the deposition of a $1 \times 1 \mathrm{~mm}^{2}$ layer of sensing material on a $2 \times 2 \mathrm{~mm}^{2}$ Aluminium Oxide substrate, and are realized through sputtering deposition, with a thickness of 10-100 nm, or by serigraphic deposition, with a thickness of about $10 \mu \mathrm{m}$.

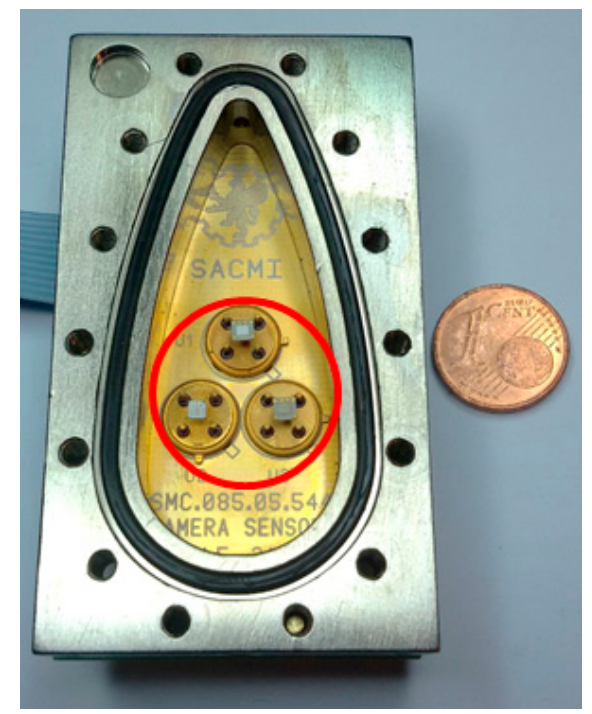

Figure 1. Open sensors chamber showing three gas sensors.

Even though this technology offers a high sensitivity to many odorous compounds and a good stability with time, several problems related to the sensor output need to be addressed to achieve a stable and reproducible instrumental response, namely:

1. Gas flow, temperature, and humidity dependence of sensor response (electrical resistance)

2. Drift of sensor baseline and sensitivity over long times (months/years), especially after inactivity times

3. Non-linear dependence of sensor response vs. gas concentration 
Many hardware and software solutions have been implemented in SACMI Electronic Noses to overcome these limitations:

1. Flow, temperature and humidity control

2. Periodical automatic calibration with reference gas

3. Acquisition of calibration curves using both reference gas and sample gas during training

As a result, these instruments can be used reliably both in laboratory for quality control measurements and outdoor for continuous environmental monitoring of odour nuisance (Figure 2). In the past years, SACMI Electronic Noses have been applied in food quality control (coffee, olive oil, tomato, etc.), packaging quality control, and monitoring of industrial activities (refineries, waste treatment plants, chemical plants, etc.).

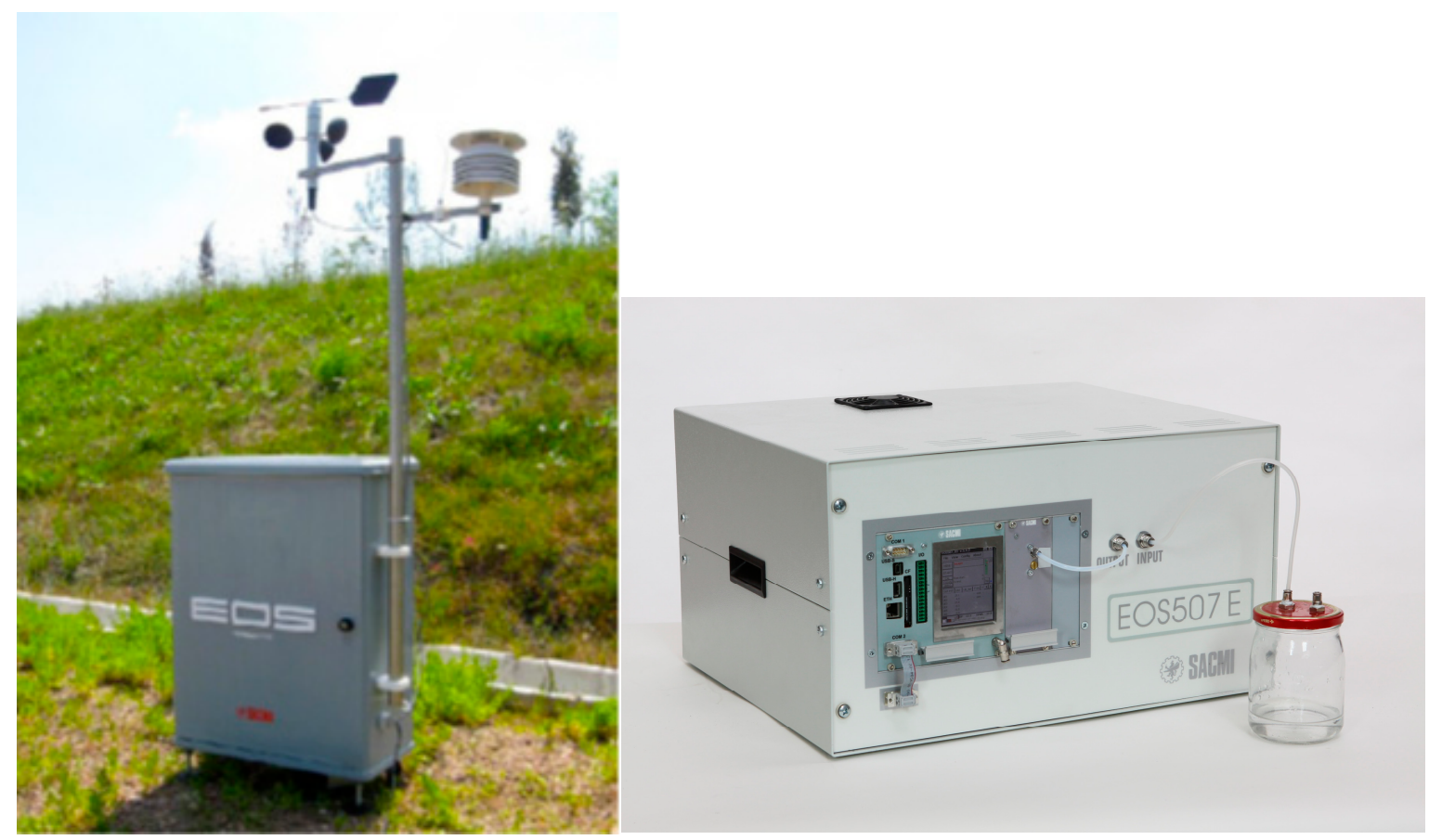

Figure 2. SACMI Electronic Olfactory Systems for outdoor (left) and laboratory (right) use.

Funding: This research received no external funding.

Acknowledgments: The authors would like to acknowledge Prof. Vincenzo Guidi (University of Ferrara) for his contribution in the development of the semiconducting metal oxides gas sensors used in SACMI Electronic Noses.

Conflicts of Interest: The authors declare no conflict of interest. The funders had no role in the design of the study; in the collection, analyses, or interpretation of data; in the writing of the manuscript, or in the decision to publish the results.

(C) 2019 by the authors. Licensee MDPI, Basel, Switzerland. This article is an open access article distributed under the terms and conditions of the Creative Commons Attribution (CC BY) license (http://creativecommons.org/licenses/by/4.0/). 